\title{
Simulation Analysis on Driving Behavior during Traffic Sign Recognition
}

\author{
Lishan Sun \\ Key Laboratory of Traffic Engineering, Beijing University of Technology \\ Beijing, 100124, P.R.China \\ Liya Yao* \\ School of Mechanical and Vehicular Engineering, Beijing Institute of Technology \\ Beijing, 100081, P.R.China \\ Jian Rong \\ Key Laboratory of Traffic Engineering, Beijing University of Technology \\ Beijing, 100124, P.R.China \\ Jinyan Lu \\ Department of Civil and Environmental Engineering, Florida International University
}

Florida, 33174, USA

Bohua Liu, Shuwei Wang

Key Laboratory of Traffic Engineering, Beijing University of Technology

Beijing, 100124, P.R.China

Received: 08-03-2011

Accepted: 19-04-2011

\begin{abstract}
The traffic signs transfer trip information to drivers through vectors like words, graphs and numbers. Traffic sign with excessive information often makes the drivers have no time to read and understand, leading to risky driving. It is still a problem of how to clarify the relationship between traffic sign recognition and risky driving behavior. This paper presents a study that is reflective of such an effort. Twenty volunteers participated in the dynamic visual recognition experiment in driving simulator, and the data of several key indicators are obtained, including visual cognition time, vehicle acceleration and the offset distance from middle lane, etc. Correlations between each indicator above are discussed in terms of risky driving. Research findings directly show that drivers' behavior changes a lot during their traffic sign recognition.

Keywords : Road safety, Driving behavior, Cognitive task, Traffic sign.
\end{abstract}

\section{Introduction}

Traffic accidents kill thousands of people every year. Whenever a driver takes his eyes off the road, even for a few seconds, he put his life and others lives at risk. The traffic signs transfer trip information to drivers through vectors like words, graphs and numbers, which contribute a lot to the distraction of driver during their recognition.

According to Stutts's research, 8.3\% drivers who were involved in the official accident in USA (1995-1999) indicated that they were distracted by foreign objects (including signs), 5.4\% said that they "looked but did not see" 1. Stutts's research strengthen the argument that traffic sign with

* Corresponding author: yaoliya@bit.edu.cn 
excessive information will affect driving behavior, and leading to risky driving.

The earliest studies in 1960s on traffic signs were related to impact assessment and cost estimation, etc ${ }^{2}$. Since $1980 \mathrm{~s}$, researchers started to focus on the driving behavior during traffic sign recognition by measuring their characteristics, reaction time, and sign recall ${ }^{3-4}$.

Effect of traffic signs on drivers has been a topic of considerable interest to researchers during the past few years. They covered a wide range of aspect related to recognition, comprehension, distraction, response, and recollection ${ }^{5-8}$. Several studies have been carried out in discussing the relationships between stress, strain, task and driving performance ${ }^{9-11}$.

Based on the above-mentioned researches, Bendak assessed the effects of roadside advertising signs on driving performance on a simulator ${ }^{12}$. Five performance indicators, including drifting from lane, recklessly crossing dangerous intersections, number of tailgating times, over-speeding and turning or changing lanes without signaling, drop consistently with the presence of advertising signs in the experiment, which indicate that the advertisement signs create a potential risk factor by distracting drivers off their original task which is driving.

Although effect of traffic signs on drivers has been widely discussed in previously researches, two important questions remain to be answered: What indicators should be selected to quantitatively reflect the correlation between recognition of traffic sign and risky driving? Furthermore, how to determine the threshold on the traffic signs with Chinese character from safety driving aspect?

This dissertation does some in-depth study of risky driving during traffic sign recognition on the driving simulator. The remainder of the paper is structured as follows. Section 2 introduces the basic methods of the experiment. Section 3 presents the indicators relate with the risky driving during traffic sign recognition and the analysis result, followed by the conclusions presented in Section 4.

\section{Methods}

\subsection{Participants}

Twenty gender-balanced participants, aged between 22 and 32, holders of a valid driving license for more than three years, were recruited in this study. All participants' vision was normal and they were required to drive for more than 30 minutes in the simulator before the test. These conditions above for inclusion in the experiment were put in order to minimize the difference between the simulation and real scene.

\subsection{Simulate scene}

One $7 \mathrm{~km}$ long two way lane road with traffic signs was simulated as shown in Figure 1. The lane, marking and traffic sign were all designed according to the Road Traffic Sign and Marking Standard of China (GB 5768-2009) ${ }^{13}$. More than 800 meters were set between each traffic sign to make sure the driver has enough time to adjust themselves before the following recognition. 4 to 8 unfamiliar location names were selected to appear on the traffic signs to ensure the authenticity of simulation test.

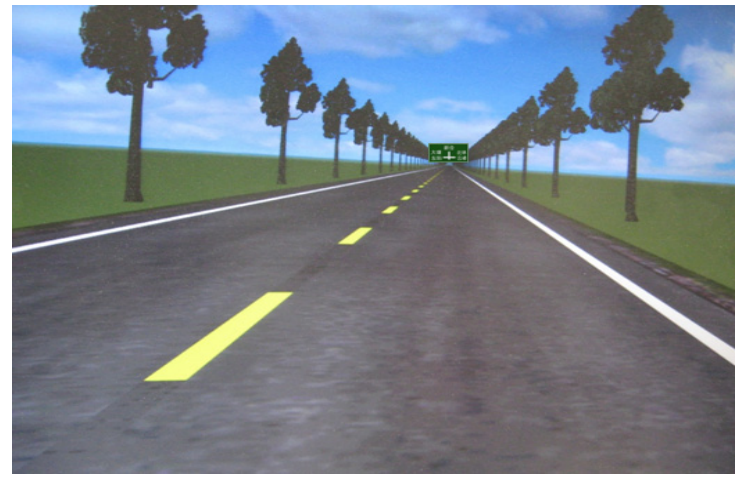

Fig.1. Simulated driving environment used in this experiment

\subsection{Driving simulator}

Driving simulator is among the most important applications of virtual reality technology. It is widely considered as valid devices for driving behavior analysis because of its low cost, safe and other significant advantages.

The driving simulator used in this study consists of four large screens and two rearview screens that 
provide a $270^{\circ}$ virtual driving environment, as shown in Fig 2. Participants were required to recognize the traffic signs at $80 \mathrm{~km} / \mathrm{h}, 100 \mathrm{~km} / \mathrm{h}$ and
$120 \mathrm{~km} / \mathrm{h}$ speeds separately, during which their fixation duration, visual cognition time, acceleration change, and driving offset, etc were recorded.

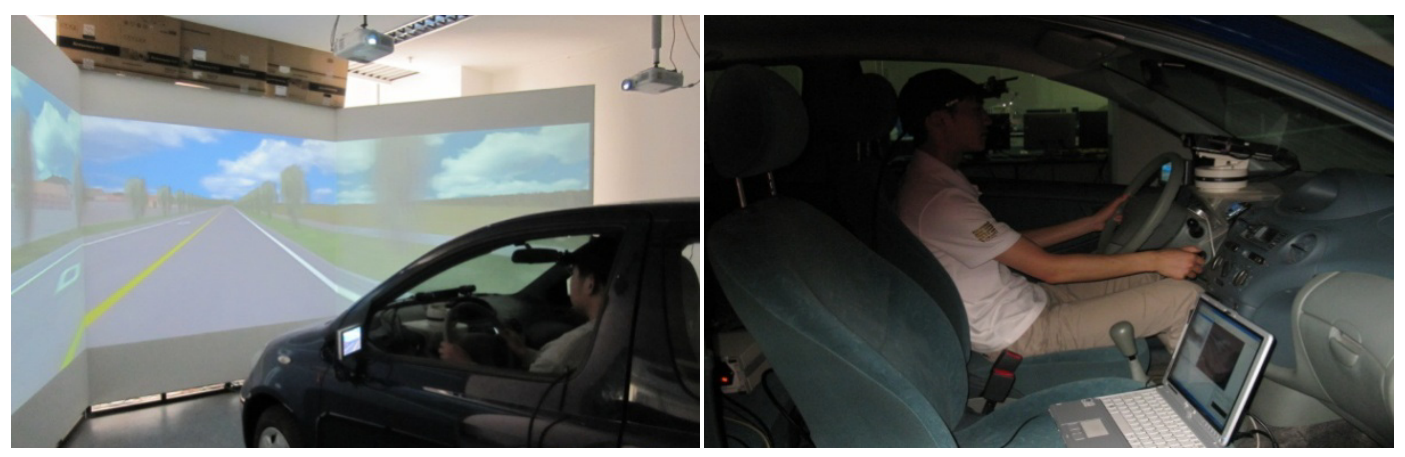

Fig.2. Driving simulator system used in this experiment

\subsection{Eye tracker}

Eye tracker was used in this study to obtain the eye movement data during the drivers' driving in the simulator, especially when they undertake the task of identifying signs.

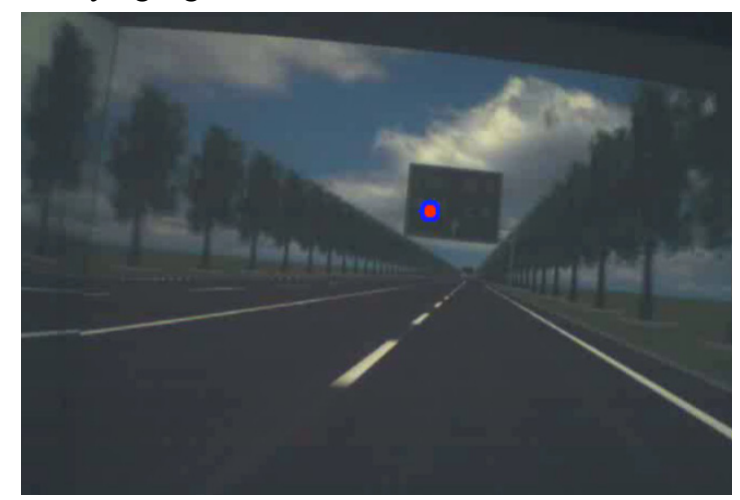

Fig.3. A screenshot from the eye tracker system

\subsection{Experimental procedure}

The whole experimental procedure can be separated into three parts as follow:

- Participants were required to read the purpose and attentions of the experiment, then 30mins were given to participants in getting familiar with the operation skills of the simulator. These steps can ensure that the participants were at their best mental state, and to obtain more accurate data.

- Participants were instructed to seat in the simulator and the eye movement system was adjusted to their heads. They were required to perform their drive task at 80 $\mathrm{km} / \mathrm{h}, 100 \mathrm{~km} / \mathrm{h}$ and $120 \mathrm{~km} / \mathrm{h}$, and to identify the target information on the traffic signs appeared in the simulated scene randomly.

- The visual cognition time, distribution of visual fixation, changes of vehicle acceleration, and offset of vehicle track will be recorded by either driving simulator or eye tracker.

\section{Data Collection and Analysis}

\subsection{Experimental data}

This study investigated how participants responded to traffic signs with different information during the simulated driving session. There are two main issues related to variable selection for driving performance analysis. The first one is the theoretical basis for variable selection. Variable selection for this model is based on other relevant studies related to driving behavior $^{14-18}$.

The other issue is the practical basis for variables select. This study is trying to analyze the driving performance during recognition from driver's perceptual and reaction aspects. Changes in driving performance, usually expressed as the driving behavior changes and the vehicle speed and trace changes. Indicators selected in this analysis should be defined to reflect most of the driver's performance during recognition as above.

Based on the two main issues considered above, the driving performance is evaluated by the fixation time, visual cognition time, acceleration, and driving offset. 
Specify of these four indicators are as follows.

Fixation time was measured from the time when the driver found the traffic sign to the time when driver identified out the necessary information and took his eye off from the traffic sign.

Visual cognition time refers to the time that driver used on the information indentify, which is usually shorter than the fixation duration.

Acceleration refers to the change of velocity, which mainly measures how fast or slow the vehicle speeds up or down during the traffic sign recognition. Velocity change can be positive, negative or zero. A "0" velocity change means that the driver performed the recognition task at a constant speed.
Driving offset refers to the distance change of the vehicle in the forward direction during the traffic sign recognition. Acceleration and driving offset are both very important in measuring the impact of traffic sign recognition on driving behavior.

In the following sections, the experiment data were discussed to estimate the relationship among the information content on traffic signs, driving speed, velocity change, and other performance indicators. Tables 1 to 4 provide a quick view of the outputs from experiment. All data were coded and analyzed using statistical software called Statistical Package for Social Sciences (SPSS, 1996).

Table 1 Visual cognition time on different information content with changed speeds

\begin{tabular}{|c|c|c|c|c|c|}
\hline \multirow{2}{*}{$\begin{array}{c}\text { Visual cognition time (s) } \\
\text { Driving speed }(\mathrm{km} / \mathrm{h})\end{array}$} & 4 & 5 & 6 & 7 & 8 \\
\cline { 2 - 6 } & 1.490 & 1.753 & 2.028 & 2.104 & 2.486 \\
\hline 80 & 1.356 & 1.602 & 1.796 & 1.734 & 1.906 \\
\hline 100 & 1.433 & 1.233 & 1.670 & 1.871 & 1.688 \\
\hline 120 &
\end{tabular}

Table 2 Fixation duration on different information content with changed speeds

\begin{tabular}{|c|c|c|c|c|c|}
\hline \multirow{2}{*}{$\begin{array}{c}\text { Fixation duration (s) } \\
\text { Driving speed }(\mathrm{km} / \mathrm{h})\end{array}$} & \multicolumn{5}{|c|}{ Information content } \\
\cline { 2 - 6 } & 4 & 5 & 6 & 7 & 8 \\
\hline 80 & 3.224 & 3.930 & 5.236 & 6.862 & 7.581 \\
\hline 100 & 3.152 & 3.787 & 4.889 & 5.591 & 6.543 \\
\hline 120 & 2.245 & 2.989 & 4.113 & 4.467 & 4.967 \\
\hline
\end{tabular}

Table 3 Acceleration on different information content with changed speeds

\begin{tabular}{|c|c|c|c|c|c|}
\hline \multirow{2}{*}{$\begin{array}{c}\text { Acceleration }\left(\mathrm{m}^{2} / \mathrm{s}\right) \\
\text { Driving speed }(\mathrm{km} / \mathrm{h})\end{array}$} & 4 & 5 & 6 & 7 & 8 \\
\cline { 2 - 6 } & 0.1735 & 0.2525 & 0.1740 & 0.2930 & 0.3147 \\
\hline 80 & 0.3277 & 0.3146 & 0.1881 & 0.3221 & 0.3660 \\
\hline 100 & 0.3882 & 0.3525 & 0.3227 & 0.5571 & 0.4033 \\
\hline 120 & &
\end{tabular}

Table 4 Driving offset on different information content with changed speeds

\begin{tabular}{|c|c|c|c|c|c|}
\hline \multirow{2}{*}{$\begin{array}{c}\text { Driving offset }(\mathrm{m}) \\
\text { Driving speed }(\mathrm{km} / \mathrm{h})\end{array}$} & 4 & 5 & 6 & 7 & 8 \\
\cline { 2 - 6 } & 0.120 & 0.202 & 0.247 & 0.266 & 0.264 \\
\hline 80 & 0.244 & 0.169 & 0.286 & 0.193 & 0.252 \\
\hline 100 & 0.256 & 0.277 & 0.249 & 0.437 & 0.292 \\
\hline 120 & &
\end{tabular}

\subsection{Validation check}

Analysis results obtained for the driving behavior during traffic sign recognition, depending on the six indicators mentioned above. Validity and correlation analysis were carried out in order to check whether the indicators value can be used for subsequent analysis.

P-P test results show that the experiment data of the six indicators follow a normal distribution (Fig.4). In addition, Table 5 shows the good correlation between 
each indicator, in which the Pearson correlation factors are all higher than 0.75 . These feasibility analyses confirm the validity of the indicators selected in this experiment.

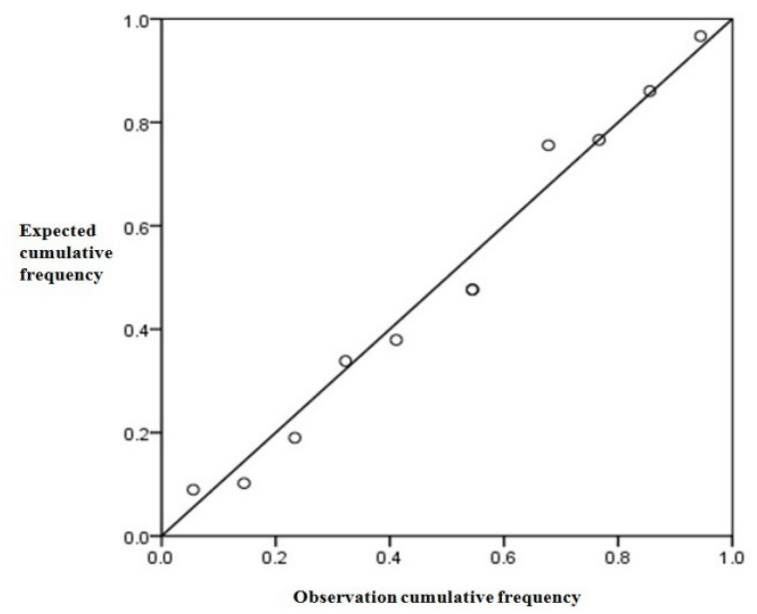

Fig.4. Normal distribution P-P test

Table 5 Pearson correlation between each indicator

\begin{tabular}{|c|c|c|c|c|c|}
\hline Pearson Correlation & $\begin{array}{c}\text { Information } \\
\text { content }\end{array}$ & $\begin{array}{c}\text { Fixation time } \\
(\mathrm{s})\end{array}$ & $\begin{array}{c}\text { Visual } \\
\text { cognition } \\
\text { time }(\mathrm{s})\end{array}$ & $\begin{array}{c}\text { Acceleration } \\
\left(\mathrm{m}^{2} / \mathrm{s}\right)\end{array}$ & $\begin{array}{c}\text { Changes of offset } \\
\text { distance }(\mathrm{m})\end{array}$ \\
\hline Information content & 1 & 0.991 & 0.986 & 0.776 & 0.906 \\
\hline Fixation time (s) & 0.991 & 1 & 0.985 & 0.773 & 0.882 \\
\hline $\begin{array}{c}\text { Visual cognition time } \\
(\mathrm{s})\end{array}$ & 0.986 & 0.985 & 1 & 0.718 & 0.891 \\
\hline Acceleration(m $/ \mathrm{s})$ & 0.776 & 0.773 & 0.718 & 1 & 0.641 \\
\hline Changes of offset & 0.906 & 0.882 & 0.891 & 0.641 & 1 \\
\hline distance $(\mathrm{m})$ & & & & & \\
\hline
\end{tabular}

\subsection{Analysis result}

Based on the validation check of indicator data, safety feature of driving behaviors during traffic sign recognition were discussed according to the changes of performance indicators.

Figure 5 shows, when driving speed increased from $80 \mathrm{~km} / \mathrm{h}$ to $120 \mathrm{~km} / \mathrm{h}$, the visual cognition time of traffic sign with the same amount of information gradually reduced. This is contrary a lot to the common sense that the higher speed the more difficult to perform recognition. In order to find out the reason, a mental status survey on participants was carried out after the simulation test. Result show that driver will be more concentrate on recognition under high driving speeds. This should be the reason why drivers could identify the necessary information on traffic sign in a relatively short time. 


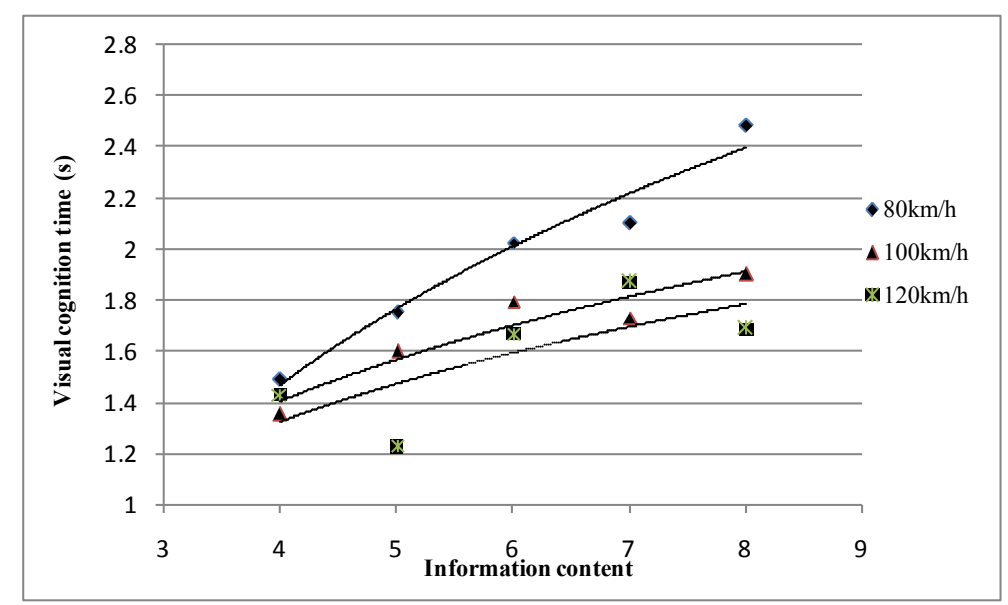

Fig.5. Correlation between visual cognition time and information content under different driving speeds

Figure 6 and 7 describe the changes in vehicle acceleration and driving offset distance in the visual recognition process. The figures show increasing trend of acceleration and driving offset when information content increases under same speed. Similarly, under same information content, with drive speed increasing, the two indicators: acceleration change and track offset distance, also show increasing trends.

There are two points worthy to be mentioned. Firstly, under the speed of $120 \mathrm{~km} / \mathrm{h}$, the acceleration change and track offset data show obvious abnormalities during driver's recognition process on traffic signs with 7 information contents. For example, the acceleration change under 7 information contents increases by about $50 \%$ compared with other amount of information. Unexpectedly, the acceleration change and track offset data of an even greater amount of information eight maintained consistency with that of four, five and six. Combined with a questionnaire on participants, traffic sign with seven information contents was considered to be overloaded. Most drivers have to take aggressive driving behavior such as deceleration in order to obtain the necessary information they want, which leading to a higher vehicle offset. For information content more than seven, the driver will be perceived that all the information can't be identified within a limited time. They have to give up some of the information, and usually do not take aggressive driving behavior. This also shows that seven should be the upper limit of information content in a separate traffic sign.

Secondly, according to figure 7 , when the speed increases from $80 \mathrm{~km} / \mathrm{h}$ to $100 \mathrm{~km} / \mathrm{h}$, tracking offset of the vehicle show reduction in average. The main reason is driver will be more focused in order to complete the identification process when their speed increased to $100 \mathrm{~km} / \mathrm{h}$. Differently, the increasing trend at $120 \mathrm{~km} / \mathrm{h}$ should be a true reflection of the exceed recognize burden on driving behavior.

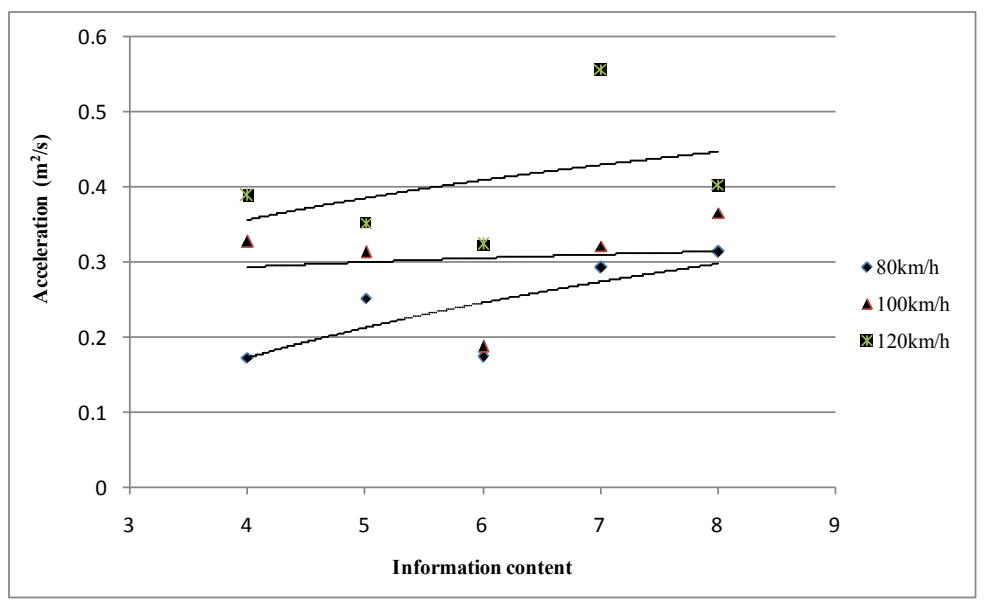

Fig.6. Correlation between Acceleration and information content under different driving speeds 


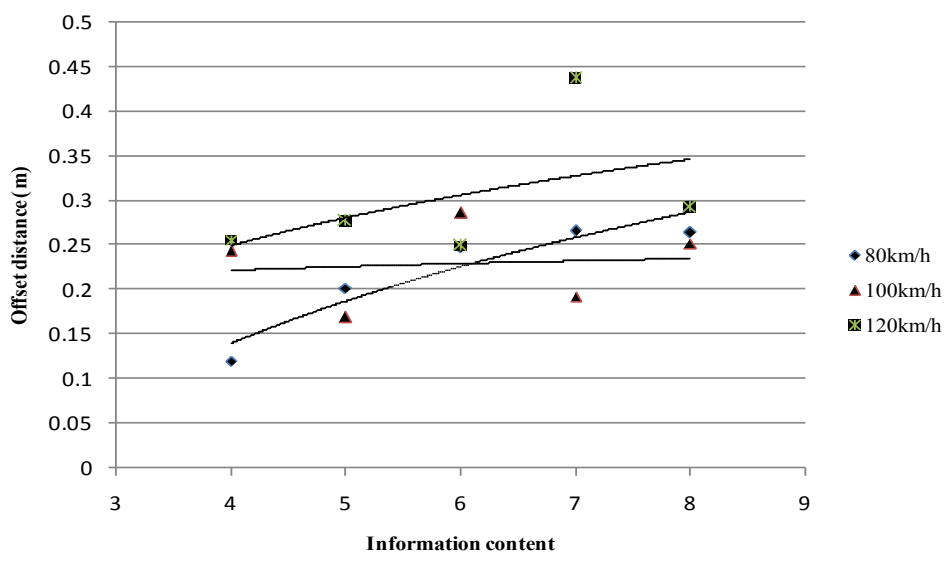

Fig.7. Correlation between driving offset and information content under different driving speeds

Figure 8 shows, with the increase of the information content on traffic sign, driver's fixation time increased more significantly than the visual cognition time. The visual cognition time remains approximately constant even the information content is eight. This result confirmed that the amount of information on traffic sign should be strictly limited, in order to make sure the driver can identify the necessary information in a very short time.

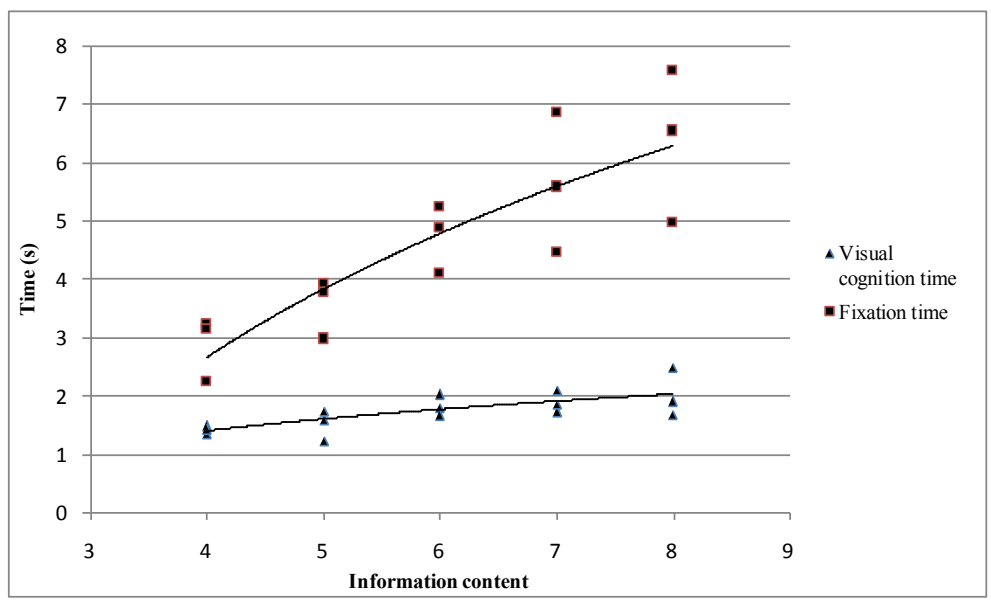

Fig.8. Correlation between cognition time and fixation time under different information content

\section{Conclusions}

Driving behavior has become an extremely important issue in enhancing the overall performance of traffic safety. In response to the absence of safety analysis on risky driving during driver's traffic sign recognition, this paper presented a factor analysis based on a dynamic simulation test

According to the data analysis of the driving simulation experiment, correlations between the acceleration, driving offset, visual cognition time, fixation time, etc, were discussed from the driving safety aspect. The main conclusions are as follows:

- Driving behavior indicators, such as cognition time, acceleration changes, track offset distance, show strong correlation with the traffic sign recognition process.

- With the increase of information content on traffic sign, the fixation time, acceleration and offset distance, etc, also show increasing trend in average accordingly. The more information contents on the traffic sign, the higher risky in driving. Seven should be the upper limit of information content in a separate traffic sign.

- Driving speed show significant effect on fixation time, acceleration and track offset while driver has to perform their recognition. Therefore, it is necessary to remind the drivers to slow down before the traffic signs with excessive information. 


\section{Acknowledgements}

The authors wish to acknowledge the financial support for this study provided by the Specialized Research Fund of Beijing Key Lab of Traffic Engineering, Beijing University of Technology (NO: JTS-2009-07); and Beijing Municipal Commission of Transport (NO: ky2009-19). Our thanks also go to other members of the research team of traffic engineering in our lab, who played a great part in the effort to collect the required data for the study.

\section{References}

1 Stutts, J.C., Reinfurt, D.W., Staplin, L., et al, The role of driver distraction in traffic crashes, Foundation for Traffic Safety, 2001, Washington, DC

2 Johansson, G., Rumar, K, Drivers and road signs: a preliminary investigation of the capacity of car drivers to get information from road signs, Ergonomics, 9 (1966), $57-62$

3 Drory, A., Shiner, D, The effects of roadway environment and fatigue on sign perception, Journal of Safety Research, 13 (1) (1982), 25-32

4 Fisher, J, Testing the effects of road traffic signs' informational value on driver behavior, Human Factors, 34 (2) (1992), 231-237

5 Hashim Al-Madani, Influence of drivers' comprehension of posted signs on their safety related characteristics, Accident Analysis and Prevention, 32 (2000), 575-581

6 Abdulbari Bener, A. Ghaffar, Abu Azab, M. Sankaran-Kutty, F. Toth and G. Lovasz, The impact of four-wheel drives on road traffic disability and deaths compared to passenger cars, Journal of the College of Physicians and Surgeons Pakistan, 16 (4) (2006), 257-260

7 Avinoam Borowsky, David Shinar, Yisrael Parmet, Sign location, sign recognition, and driver expectancies, Transportation Research Part F, 11 (2008), 459-465

8 Wuhong Wang, Fuguo Huo, Huachun Tan, et al, A framework for function allocation in intelligent driver interface design for comfort and safety, International Journal of Computational Intelligence Systems, 3(5) (2010), 531-541

9 Torbjorn Rundmo, Hilde Iversen, Risk perception and driving behavior among adolescents in two Norwegian counties before and after a traffic safety campaign, Safety Science, 42 (2004), 1-21

10 Wolfgang Fastenmeier, Herbert Gstalter, Driving task analysis as a tool in traffic safety research and practice, Safety Science, 45 (2007), 952-979

11 J.K. Caird, S.L. Chisholm, J. Lockhart, Do in-vehicle advanced signs enhance older and younger drivers' intersection performance? Driving simulation and eye movement results, International Journal of Human-Computer Studies, 66 (2008), 132-144

12 Salaheddine Bendak, Khalid Al-Saleh, The role of roadside advertising signs in distracting drivers, International Journal of Industrial Ergonomics, (2010), $1-4$

13 GB 5768-2009, Road traffic signs and markings, China

14 Hashim Al-Madani, Abdul-Rahman Al-Janahi,
Assessment of drivers' comprehension of traffic signs based on their traffic, personal and social characteristics, Transportation Research Part F, 5 (2002), 63-76

15 Shunhui Chang, Chihyung Lin, Chinping Fung, et al, Driving performance assessment: Effects of traffic accident location and alarm content, Accident Analysis and Prevention, 40 (2008), 1637-1643

16 Tuncay gurbuz, Multiple criteria human performance evaluation using choquet integral, International Journal of Computational Intelligence Systems, 3(3) (2010), 290-300

17 Narayanan NK, Kabeer V, Face Recognition Using Nonlinear Feature Parameter and Artificial Neural Network, International Journal of Computational Intelligence Systems, 3(5) (2010), 566-574

18 Juan Carlos Augusto, Hui Wang, Jun Liu, Situation assessment in disaster management, International Journal of Computational Intelligence Systems, 1(3) (2008), 237-247 